\title{
NetGen: a novel network-based probabilistic generative model for gene set functional enrichment analysis
}

\author{
Duanchen Sun ${ }^{1,2,3}$, Yinliang Liu ${ }^{1,2,3}$, Xiang-Sun Zhang ${ }^{1}$ and Ling-Yun $\mathrm{Wu}^{1,2,3^{*}}$ \\ From The 10th International Conference on Systems Biology (ISB 2016) \\ Weihai, China. 19-22 August 2016
}

\begin{abstract}
Background: High-throughput experimental techniques have been dramatically improved and widely applied in the past decades. However, biological interpretation of the high-throughput experimental results, such as differential expression gene sets derived from microarray or RNA-seq experiments, is still a challenging task. Gene Ontology (GO) is commonly used in the functional enrichment studies. The GO terms identified via current functional enrichment analysis tools often contain direct parent or descendant terms in the GO hierarchical structure. Highly redundant terms make users difficult to analyze the underlying biological processes.

Results: In this paper, a novel network-based probabilistic generative model, NetGen, was proposed to perform the functional enrichment analysis. An additional protein-protein interaction (PPI) network was explicitly used to assist the identification of significantly enriched GO terms. NetGen achieved a superior performance than the existing methods in the simulation studies. The effectiveness of NetGen was explored further on four real datasets. Notably, several GO terms which were not directly linked with the active gene list for each disease were identified. These terms were closely related to the corresponding diseases when accessed to the curated literatures. NetGen has been implemented in the R package CopTea publicly available at GitHub (http://github.com/wulingyun/CopTea/).

Conclusion: Our procedure leads to a more reasonable and interpretable result of the functional enrichment analysis. As a novel term combination-based functional enrichment analysis method, NetGen is complementary to current individual term-based methods, and can help to explore the underlying pathogenesis of complex diseases.
\end{abstract}

Keywords: Gene ontology, Enrichment analysis, Network-based probabilistic generative model, Integer programming, Complex diseases

\section{Background}

High-throughput experimental techniques, such as microarray, mass spectrometry and next-generation sequencing, have become indispensable tools for biological and medical researches. These high-throughput experiments usually generate large interesting gene lists as their final outputs, which share some certain characteristics. A large

\footnotetext{
* Correspondence: lywu@amss.ac.cn

${ }^{1}$ Institute of Applied Mathematics, Academy of Mathematics and Systems Science, Chinese Academy of Sciences, Beijing 100190, China

${ }^{2}$ National Center for Mathematics and Interdisciplinary Sciences, Chinese Academy of Sciences, Beijing 100190, China

Full list of author information is available at the end of the article
}

fraction of the gene outputs specifies the key biological functions underlying the studied samples. Therefore, interpreting the biological meaning of the similar characteristics and exploring the functional relationships among the selected genes are one of the important and challenging tasks in bioinformatics.

Gene Ontology (GO) project is a major bioinformatics initiative to produce a structured, dynamic, controlled vocabulary to describe key domains of molecular and cellular biology [1] and unify the representation of gene and gene product attributes [2, 3]. Due to the hierarchical structure of the GO, terms located at the top region often have more general molecular and cellular interpretation, 
and cover larger genes. On the other hand, terms at the bottom of the hierarchical structure represent more specific biological explanations. For example, 'induction of apoptosis' is a part of 'apoptosis', and the former is the most specific term. Therefore, genes annotated by the specific term are implicitly annotated by both its parent terms. As consequence, we cannot clearly know that which child term is a major reason to make the gene set significant, if the term 'apoptosis' is determined to be significantly enriched. So if the significant terms are chosen based on enrichment $p$-values, such as commonly used statistical methods, they may obscure other more important terms and make it hard to determine the most relevant explanations.

For addressing this issue, a variety of methods, aiming at finding the most involved functional relationships among the selected genes, have been developed during the past decades to perform the GO enrichment analysis. Basing on the model input (type of gene list) and the output (evaluation pattern of the identified GO terms), these methods can be briefly categorized into three classes (See Additional file 1, Table S1, Class I-III). The first class, represented by GOMiner [4], EASE [5], GOstat [6] onto-express [7], EnrichNet [8], MAPPFinder [9], etc., used a gene set as model input and output the significant level of each GO term, which was mainly based on the Fisher's exact test [10]. A preset threshold value was usually selected by user to generate the gene list of interest. The second class, represented by GSEA [11], GLOBALTEST [12], SIGPATHWAY [13], PAGE [14], SAFE [15], EasyGO [16], etc., used a whole gene list with corresponding scores to evaluate each GO term. These methods combined the selection of differential expression genes and the enrichment analysis, and no need to preset a threshold for generating the gene list of interest. The major disadvantage of class II methods is its lacking of flexibility, for the scores are not easily accessible under sophisticated biological experiments. Class I and II both individually evaluated the terms, which brought a higher similarity and redundancy of identified terms. To make up this drawback, the third class, represented by DAVID [17], MGSA [18], GenGO [19], MCOA [20], eliminated the redundancy of enrichment analysis from the perspective of term set. Given a gene list as model input, one or more most enriched term sets were returned as model outputs. The terms in a term set may be similar or complementary. For example, DAVID [17] reduces the redundancy in the result of enrichment analysis by grouping similar terms into functional clusters.

In this paper, a novel network-based probabilistic generative model, NetGen, was proposed to perform the enrichment analysis. We followed the framework introduced in GenGO [19] that the gene list of interest was generated by some $\mathrm{GO}$ terms, which can retell the true story beneath the biological experiment. Lu, et al., mainly assumed that the active information of terms could passed to the annotated genes. Therefore, they defined a probabilistic model on the activation graph which contains both gene and GO nodes. By maximizing the likelihood of their model conditioned on the set of active genes, their final results shown that GenGO is prone to directly identify the combination of complementary (i.e. non-redundant) terms, and GenGO has a good performance when compared with other methods on both yeast and human GO database. Particularly, we provided a brand new perspective to consider this framework. In our model, an additional protein-protein interaction (PPI) network was explicitly used to assist the functional analysis. We assumed that the effect of active terms not only passed to the directly annotated genes, but also can affected the neighboring genes of the annotated genes in the PPI network. This supporting influence get weaker when the distances from the annotated genes get larger. Our procedure can lead to a more reasonable and explainable result of the functional analysis. Maximizing the log-likelihood estimation function can be formulated as a $0-1$ integer programming problem. We used a greedy algorithm to identify the most enriched term combination.

During the past years, many methods that integrate the information of biological network have been developed to improve the performance of the enrichment analysis. For example, Wang, et al. [21] proposed a network ontology analysis (NOA) method to perform the GO enrichment analysis on biological networks. Network enrichment analysis (NEA) [22] extends the traditional overlap statistic in gene-set analysis to network links between genes in experimental output list and those in function terms. EnrichNet [8] first scores the distances between the gene list and reference gene set in the network using random walk with restart algorithm, then compares these scores with a background model to derive their final results. There are two reasons why we added the network information to assist the functional analysis. First, the GO annotation database is far from complete. Due to the underlying incompletion of GO annotation, some annotation links between GO terms and genes have not been established. Therefore, traditional functional analysis cannot identify these candidate terms. On the other hand, the interaction network was built based on the physical contacts of proteins. Proteins are prone to share a similar biological function, if their distance is short in the PPI network, which can be used to compensate for the incompletion of the GO annotation. Second, with the additional network information, our network-based generative model can simulate the upstream-downstream regulatory mechanism. Specifically, the neighboring genes and the directly annotated genes can be viewed as the downstream targets and the 
upstream regulators, respectively. In many cases, upstream regulators have only subtle expression variation therefore they may not be directly identified and emerge in the gene list of interest. However, through the directly annotated genes and network, the effect of active terms passes to the downstream genes, which may be observed and selected into the gene list of interest. The potential terms can be identified with a more reasonable and explainable result.

In this work, we first compared the performance of NetGen and classic individual term-based or term combination-based enrichment analysis methods in the simulation studies. NetGen achieved a superior performance than GenGO [19] and Fisher's exact test [10], when the active gene list was generated under our assumption. We further explored the effectiveness of NetGen on four real datasets. Notably, we identified several terms which were not directly linked with the active gene list (Fisher's exact test, $p=1$ ) for each disease. These terms were closely related to the corresponding diseases according to the literature. All these pieces of evidence showed that NetGen is an efficient computational tool for functional enrichment analysis and can help to explore the underlying pathogenesis of complex diseases. NetGen has been compiled in the R package CopTea, which is available at GitHub (http://github.com/ wulingyun/CopTea/) for users.

\section{Methods}

\section{Network-based probabilistic generative model}

In our network-based probabilistic generative model, the model input is the gene list of interest $G$ (active gene list). We would like to identify the most enriched GO term set, which provides a reasonable biological explanation to $G$. Here our model assumed that $G$ is generated by several unknown active GO terms, by which we can investigate and gain the insights into the related biological experiments or problems. Under this assumption, we completely modeled the generative process that propagate the active information from terms to genes and further through the biological network. In detail, our generative model can be explicated as follows.

First, some related GO terms are activated under the specific biological condition. The genes which are annotated by these active GO terms are defined as the core genes. Each core gene is activated (i.e. observed in biological experiments result $G$ ) with a relatively large probability $p_{1}$. Second, we explicitly take the information propagation in biological network into consideration. Two genes with a relationship in biological network prone to have the similar functions. Generally speaking, the larger distance between two genes, the lower probability that they share the same biological functions (i.e. they are annotated by the same term). Therefore, we assumed that the genes which are close to the core genes in biological network do have a small chance to be activated, and the influences of core genes get weaker with the distances increase. In this paper, we only consider the direct neighbors of the core genes in biological network, which are defined as the peripheral genes. Each peripheral gene is activated with a relatively low probability $p_{2}$. Last, due to the inevitable noises and errors in biological experiments, other genes also have a very low probability $q$ to be picked up into the active gene list.

Intuitively, we can interpret this process in terms of a tripartite graph, which can represent the relationship between GO terms, core genes, peripheral genes and other genes on the biological network (Fig. 1). Given the set of active GO terms, the core genes (red nodes in Fig. 1) can be identified immediately based on GO annotation. According to the generative procedure mentioned above, by using the information of biological network, the peripheral genes, represented as blue nodes in Fig. 1, can also be found out. All the remaining genes are other genes, which are represented as gray nodes in Fig. 1. Three types of genes are selected into the active gene list by probabilities, $p_{1}, p_{2}, q$, respectively, where $p_{1}>p_{2} \gg q$.

Note that though we all use the nomenclature "active" to describe the selected terms and genes, their underlying meanings are different. As shown in Fig. 1 and Fig. 2, the active genes are the model input whereas the active terms are the variables which need to be inferred as model output.

\section{Maximum likelihood estimation problem}

Given an active gene list $G$, we infer the unknown active GO terms by maximizing the likelihood of observing the data. First, we give the following definitions as illustrated in Fig. 1:

(i) $\mathrm{N}_{1}$ : Node set of active core genes.

(ii) $E_{1}$ : Edge set from active $\mathrm{GO}$ terms to inactive core genes.

(iii) $\mathrm{N}_{2}$ : Node set of active peripheral genes.

(iv) $E_{2}$ : Edge set from core genes to inactive peripheral genes.

(v) $O_{A}$ : Node set of other active genes.

(vi) $O_{I}$ : Node set of other inactive genes.

Using these symbols, we defined the following loglikelihood function:

$$
\begin{aligned}
L\left(G \mid C, p_{1}, p_{2}, q\right) & =\left|N_{1}\right| \log p_{1}+\left|E_{1}\right| \log \left(1-p_{1}\right) \\
& +\left|N_{2}\right| \log p_{2}+\left|E_{2}\right| \log \left(1-p_{2}\right) \\
& +\left|O_{A}\right| \log q+\left|O_{I}\right| \log (1-q)-\alpha|C|
\end{aligned}
$$

where $C$ denotes the set of inferred active GO terms and $G$ is the observed active gene list. This log-likelihood 


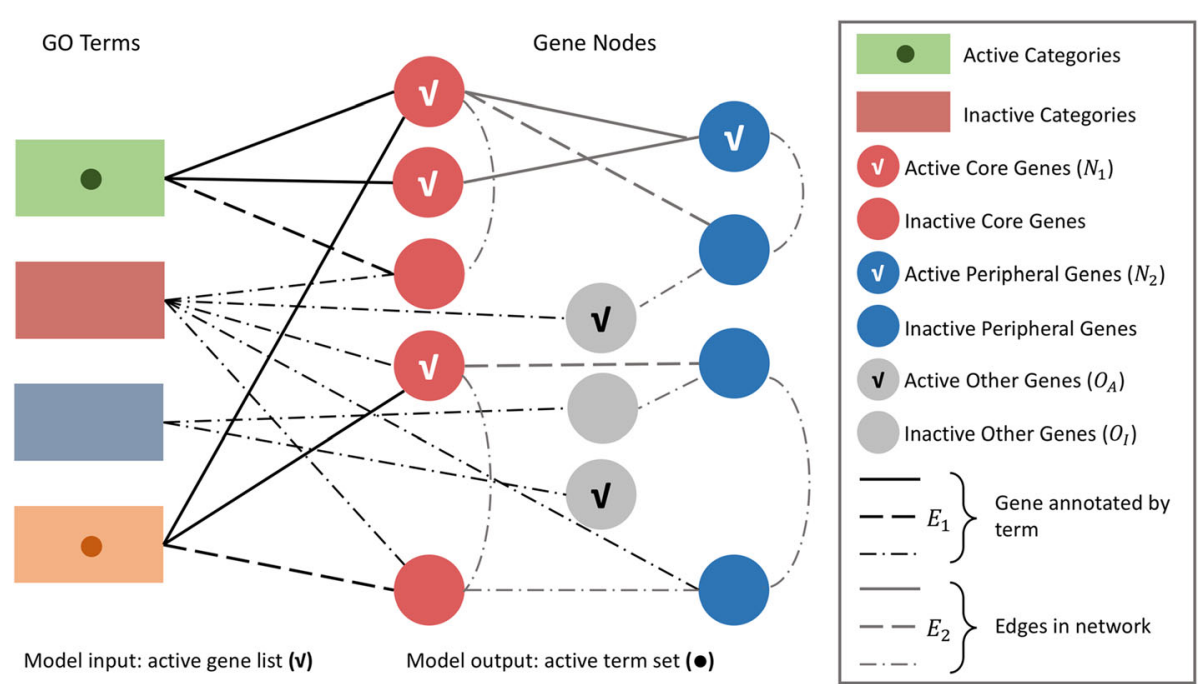

Fig. 1 The tripartite graph for an intuitive interpretation of the generative process. Rectangular nodes represent the GO terms, and rounded nodes represent all genes annotated in one species. An edge is introduced between a GO term and a gene node, if and only if the gene is annotated by that term. The edges between two gene nodes are the counterpart edges in a biological network. The different line types represent different connection types: active-active, active-inactive, inactive-inactive

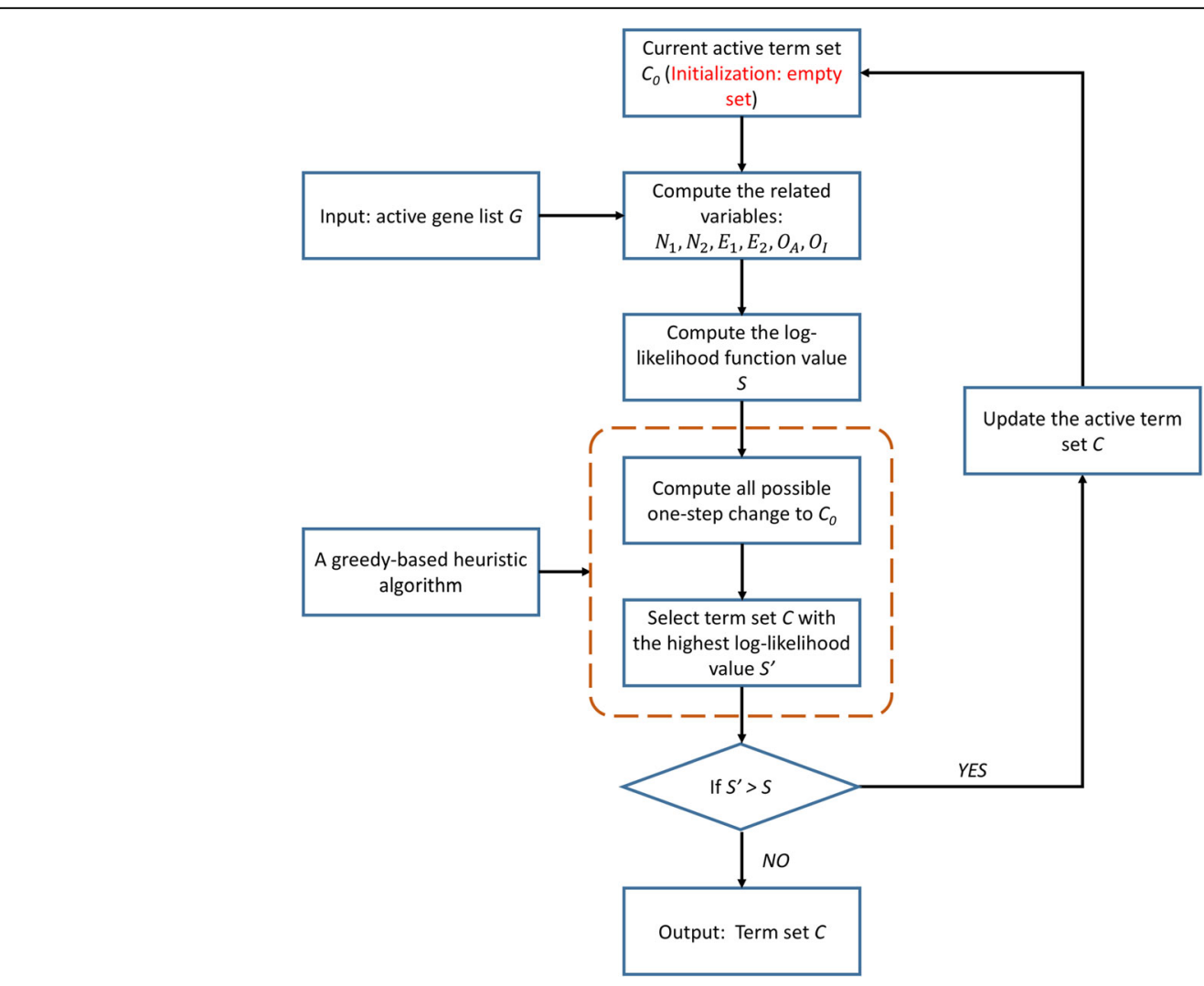

Fig. 2 The workflow of NetGen. The active gene list $G$ is the model input. We want to identify the most enriched GO term set $C$, which has a reasonable biological explanation to $\mathrm{G}$, as the final output. A greedy-based heuristic algorithm was used to maximum the log-likelihood function 
function captures the generative property as we described above. First, the core genes had a relatively larger probability $p_{1}$ to be active, and the peripheral genes had a relatively lower probability $p_{2}$ to be active. Second, we penalized the inactive core genes and the inactive peripheral genes using the size of edge sets $E_{1}$ and $E_{2}$ instead of the size of node sets, respectively. In this way, we can reduce the redundancy of the active GO terms. Third, all the other genes both had the smallest probability $q$ to be activated by external factors, such as noise, uncontrollable error in experiments, the incompletion of GO annotation and so on. In the end, a penalty term $|C|$ was added in the likelihood function, so that the model will prefer a smaller set of active GO terms to facilitate the interpretation of the active gene list, and the parameter $\alpha$ was a positive number to balance the log-likelihood and the penalization term. The maximum likelihood estimation (MLE) approach was used for the functional enrichment analysis to identify the most enriched terms. In fact, maximizing the above log-likelihood function is equivalent to minimize the difference between the active gene list and the core and peripheral nodes activated by inferred active terms.

\section{Integer programming model and greedy algorithm}

The above maximum likelihood estimation problem for searching the best GO term set $C$ can be formulated as an integer quadratic programming (IQP) model. Supposing there are $M$ GO terms and $N$ genes, the annotations are denoted by an annotation matrix $A$, with dimension $M \times N$. The element $A_{i j}=1$, if the gene $j$ is annotated by the term $i$, and $A_{i j}=0$ otherwise. An active gene list is given by the vector $g$ of length $N$. The element $g_{i}=1$, if the gene $j$ is in the active gene list, and $g_{i}=0$ otherwise. The $N \times N$ matrix $B$ is the adjacent matrix of biological network. The element $B_{i j}=1$, if the gene $i$ is connected to the gene $j$ in network, and $B_{i j}=0$ otherwise. The IQP model is formulated as follows:

$$
\begin{aligned}
\max _{x, y, z} \sum_{j} y_{j} g_{j} \log p_{1} & +\sum_{i, j} x_{i} A_{i j}\left(1-g_{j}\right) \log \left(1-p_{1}\right) \\
& +\sum_{j}^{j} z_{j} g_{j} \log p_{2} \\
& +\sum_{i, j} y_{i} B_{i, j}\left(1-y_{j}\right)\left(1-g_{j}\right) \log \left(1-p_{2}\right) \\
& +\sum_{j}\left(1-y_{j}\right)\left(1-z_{j}\right) g_{j} \log q \\
& +\sum_{j}\left(1-y_{j}\right)\left(1-z_{j}\right)\left(1-g_{j}\right) \log (1-q) \\
& -\alpha \sum_{i} x_{i}
\end{aligned}
$$

$$
\begin{array}{rll}
\text { s.t. } & y_{j} \leq \sum_{i} A_{i j} x_{i} & j=1,2, \cdots, N \\
y_{j} \geq A_{i j} x_{i} & i=1,2, \cdots, M ; j=1,2, \cdots, N \\
z_{j} \leq \sum_{i} B_{i j} y_{i} & j=1,2, \cdots, N \\
z_{j} \geq B_{i j} y_{i} & i=1,2, \cdots, N ; j=1,2, \cdots, N \\
x_{i} \in\{0,1\} & i=1,2, \cdots, M \\
y_{j}, z_{j} \in\{0,1\} & j=1,2, \cdots, N
\end{array}
$$

The binary variable $x_{i}$ denotes whether the term $i$ is selected in the final output (i.e. active). $x_{i}=1$ means that the term $i$ is active, and $x_{i}=0$ otherwise. The binary variable $y_{j}$ denotes whether the gene $j$ is a core gene, i.e. annotated by at least one active term. $y_{j}=1$ if gene $j$ is a core gene, and $y_{j}=0$ otherwise. The binary variable $z_{j}$ denotes whether the gene $j$ is a peripheral gene. $z_{j}=1$ if gene $j$ is a peripheral gene, and $z_{j}=0$ otherwise. Using these symbols, the sizes of $N_{1}, E_{1}, N_{2}, E_{2}, O_{A}, O_{I}$ defined in MLE can be calculated as the corresponding items in the objective function of IQP. Besides, the constraints imply the basic assumptions in our generative model. $y_{j} \leq \sum_{i} A_{i j} x_{i}$ and $y_{j} \geq A_{i j} x_{i}$ restrict that only the genes linked with at least one active term can be a core gene. $z_{j} \leq \sum_{i} B_{i j} y_{i}$ and $z_{j} \geq B_{i j} y_{i}$ restrict that only the genes linked with at least one core gene in network can be a peripheral gene.

The above IQP is difficult to solve exactly since integer programming generally is NP-hard, from the perspective of computational complexity. It may not be applicable for real annotation data analysis, which make us turn to use a heuristic algorithm to seek for an approximate solution. As in GenGO [19], we used a greedy algorithm to find the near optimal set of GO terms. Briefly, the algorithm first finds out a single term whose loglikelihood function value is the highest. Then in each iteration, the algorithm considers all possible one-step changes of the current set of active terms, i.e. adding or deleting one term each time. It records the term that make the largest improvement to the current loglikelihood function value, and consequently updates the current term set. The algorithm stops if the loglikelihood function value cannot be further improved by any one-step change.

In conclusion, the whole workflow of NetGen is shown in Fig. 2. First, using the active gene list $G$ and the current active term set $C_{0}$, determine the core and peripheral genes and compute the related numbers $N_{1}, N_{2}$, $E_{1}, E_{2}, O_{A}, O_{I}$. Second, compute the log-likelihood function value $S$ of the current active term set $C_{0}$. Third, using a greedy-based heuristic algorithm to obtain an alternative updated active term set $C$. Last, update the current term set $C_{0}$ iteratively until no improvement 
could be achieved by any one-step change and then output the final term set.

\section{Mixed parameter selection strategy}

The selection of model parameters is important and difficult. When applying NetGen in practical applications, in fact, the true parameters for generating the active gene list is largely unknown, and the inappropriate parameter selection may affect the performance of enrichment analysis. To alleviate the effects of parameter selection, parameter sensitivity analysis (see Additional file 1) was performed first to test the robustness of NetGen parameters. According to the helpful information supplied from the sensitivity analysis, we designed a mixed parameter selection strategy to facilitate the use of NetGen in real data analysis.

Given a list of active genes, the following mixed parameter selection strategy was performed to produce multiple solutions, which can offer more information to the biologist for downstream analysis.

1. The candidate values for model parameters were fixed as $p_{1}=0.8$ or $0.5, p_{2}=0.1$ or 0.05 , $q=0.01$ or $0.001, \alpha=3$.

2. Run NetGen algorithm using all eight combinations of candidate parameter values. An active term set was obtained for each parameter combination.

3. Union the genes annotated by at least one term in the active term set to form a super pseudo term for each parameter combination.

4. For each super pseudo term, compute the enrichment $p$-value using the Fisher's exact test.

5. Output the results of all eight parameter combinations in ascending order of $p$-values.

As for GenGO, the model is unrelated to the parameter $p_{2}$. All four parameter combinations $\left(p_{1}=0.8\right.$ or 0.5 , $q=0.01$ or $0.001, \alpha=3$ ) were used to perform the same mixed parameter selection strategy. Ultimately, the results of four parameter combinations are output in ascending order of $p$-values.

\section{Simulated datasets}

In our study, we first tested the effectiveness of NetGen via simulation studies, on the biological process (BP), the molecular function (MF) and the cellular component (CC) domains, respectively. Two other alternative methods, GenGO [19] and Fisher's exact test [10], were also taken into consideration for comparison. Here, we used the following four groups of simulation parameters (generating parameters) to generate the related active gene list:

1) $p_{1}=0.8, p_{2}=0.3, q=0.001, \alpha=3$
2) $p_{1}=0.5, p_{2}=0.3, q=0.001, \alpha=3$

3) $p_{1}=0.8, p_{2}=0.1, q=0.001, \alpha=3$

4) $p_{1}=0.8, p_{2}=0.3, q=0.01, \alpha=3$

The whole workflow of simulation studies is as follows:

1. We restricted the terms in one domain (BP, $\mathrm{CC}$ or $\mathrm{MF}$ ), whose number of covered gene was 2 to 500 (to remove the terms too specific or too general), and then randomly selected 500 terms 10 times from this refined term set to obtain 10 annotation sets.

2. In each annotation set, we randomly selected 5 terms 20 times as the target active term set. For each target active term set, we generated the active gene list using a fixed generating parameter combination.

3. Each active gene list was used as the model input. The solving parameter values of NetGen were the same as the generating parameter values. Since GenGO is unrelated to parameter $p_{2}$, we only used the values of $p_{1}, q, \alpha$ to obtain the output terms.

4. The 200 model outputs were combined to obtain a $2 \times 2$ contingency table. Besides, the Bonferroni corrected hypergeometric test $p$-values were used as the significant scores for these output terms.

5. The precision-recall curves were plotted to test the performance of each method.

For each generating parameter combination, we plot a precision-recall curve, on which each point corresponds to a cutoff of corrected hypergeometric test $p$-value. The precision and recall are defined as:

$$
\begin{aligned}
\text { precision } & =\frac{T P}{T P+F P} \\
\text { recall } & =\frac{T P}{T P+F N}
\end{aligned}
$$

where $T P, F P$ and $F N$ are the abbreviations for true positive, false positive and false negative. $T P$ is the number of true active terms below the cutoff. $F P$ stands for the number of inactive terms below the cutoff. $F N$ is the number of active terms above the cutoff. We set the significant scores of terms that are not in the model output to 1 , for ensuring the correct calculation of recall.

\section{Real datasets}

In our study, we used two kinds of data, GO annotation and PPI network, to identify the active terms via NetGen.

The GO annotation was extracted from R package org. $H$ s.eg.db in Bioconductor project. The detailed information 
about the GO annotation data was summarized in Additional file 1 Table S4.

PPI data was extracted from Human Protein Reference Database (HPRD, http://www.hprd.org/) [23]. After removing the multiple edges and the self-loops, the refined PPI network contained 9453 genes and 36,867 interactions. In our model, we did not restrict our analysis on the overlapped genes (i.e. genes included in the PPI network and annotated by at least one GO term). Instead, we used the network information of the overlapped core genes to assist our model to identify the enriched terms.

To test the performance of NetGen in real data applications, four microarray gene expression datasets of human complex diseases were selected from the Gene Expression Omnibus repository (accession number GSE4115, GSE11223, GSE9750, GSE36895, respectively), basing on several criteria (see Additional file 1). After the preprocessing of the original datasets, we sorted the microarray genes by ascending order of the $p$-values derived by the Student's t-test on the disease and control samples. The top 100 genes were selected as the differential expression gene set. The differential expression gene set was then overlapped with the annotated genes, which were used as the final active gene list to perform the enrichment analysis.

\section{Semantic similarity based analysis}

The GO semantic similarity has been widely used in the field of bioinformatics. It provides a criterion to measure the redundancy between the functional terms. Generally speaking, a lower semantic similarity score indicates a lower redundancy between two GO terms. In this paper, we used the averaged GO semantic similarity score to measure the redundancy of the identified terms. The averaged GO semantic similarity score is defined as:

$$
\operatorname{ASS}(S)=\left(\begin{array}{l}
n \\
2
\end{array}\right)^{-1} \sum_{1 \leq i<j \leq n} \operatorname{score}\left(S_{i},, S_{j}\right)
$$

where $S=\left\{S_{1}, S_{2}, \cdots, S_{n}\right\}$ is the identified term set and $n$ is the size of $S$. $\operatorname{score}\left(S_{i}, S_{j}\right)$ is the semantic similarity score between GO terms $S_{i}$ and $S_{j}$. To make the result more comparable, a background distribution of the averaged semantic similarity scores was derived on each dataset, which was obtained by randomly resampling

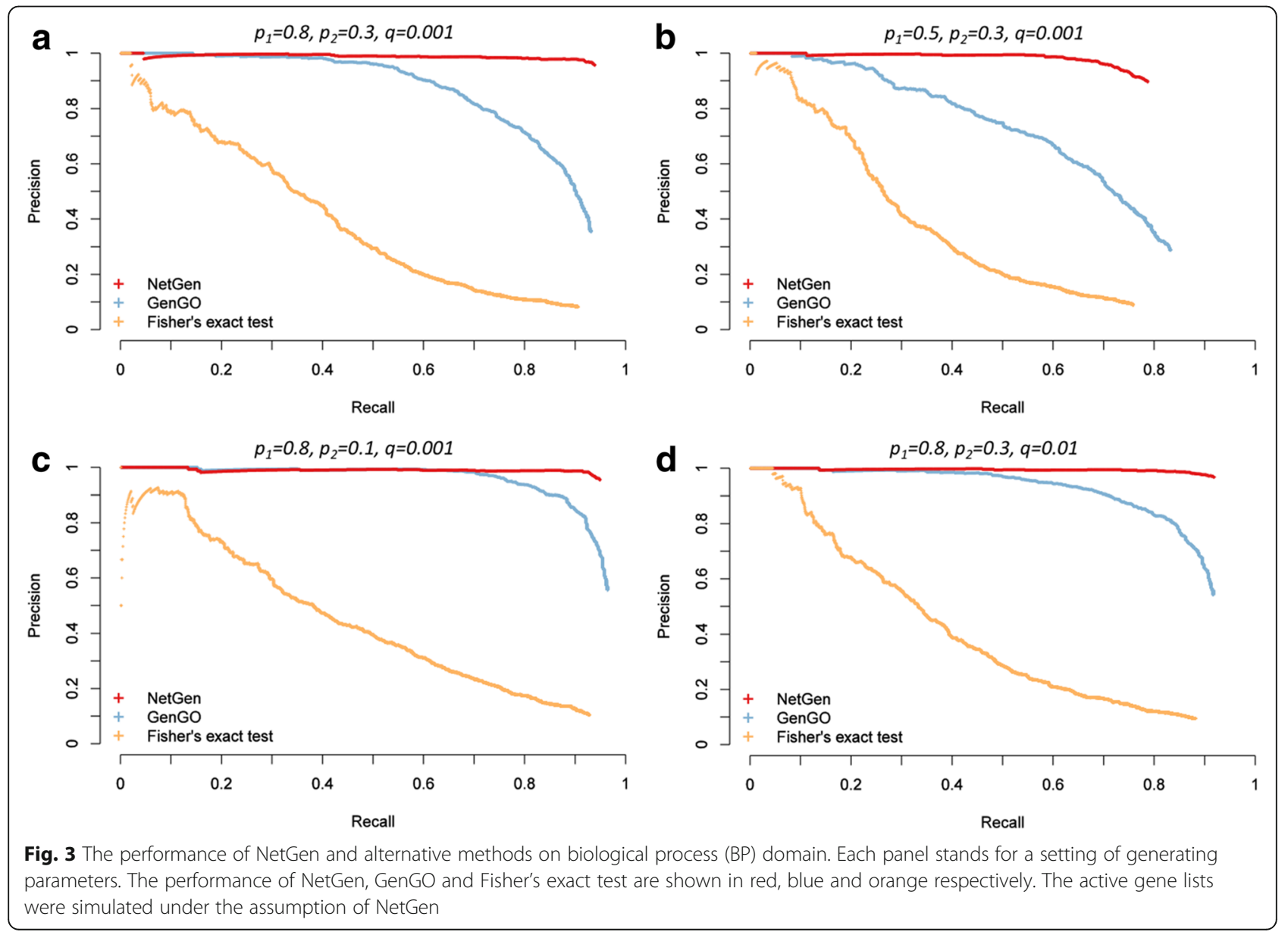


term sets with same size for 100,000 times. As for the Fisher's exact test, the most enriched top $n$ terms were selected. $n$ is the term set size identified via NetGen. In this work, the semantic similarity score was computed using the R package GOSemSim [24], which is compiled in Bioconductor [25].

\section{Results}

\section{Simulation studies}

We first test the performance of NetGen via simulation studies. The detailed description of our simulation study can be found in Methods. The results on biological process, cellular component and molecular function domain are shown in Fig. 3, Additional file 1 Fig. S5 and S6, respectively.

From the results, we can see that NetGen outperformed other alternative methods on both three domains, and showed a more stable performance when using all kinds of parameter combinations. Fisher's exact test is independent of the term combinations and neglects the overlap of parent or descendant terms. Consequently, an inferior performance was observed since highly redundant terms were identified. The performance of GenGO was closely related to the selection of generating parameter $p_{1}$ and $p_{2}$, when the true active gene list is explicable using network information. In detail, the performance of GenGO significant decreased, when the impact of network becomes larger, i.e. $p_{2} / p_{1}$ increased (Fig. 3, Fig. S5-S6 B). A comparable performance was observed, if the original active gene list was less impact by the network information, i.e. $p_{2} / p_{1}$ decreased (Fig. 3, Fig. S5-S6 C). Besides, increasing probability $q$, the influence of noise or other uncontrollable error in experiment, can improve the performance of GenGO (Fig. 3, Fig. S5-S6 D), which was accordance with the sensitivity analyses of $q$ (see Fig. S3). This may be explained by that the noise itself can offset the effect of active genes generated via network propagation.

In addition to the above simulation procedure, we also simulated another alternative circumstance. We wonder how the performance of NetGen behaved, if the active gene lists were actually unrelated to the network information. Therefore, active gene lists were generated under the assumption of GenGO model, i.e. $p_{2}=0$. The performance and discussion can be found in Additional file 1 (Figure S7-S9), which showed that NetGen with small $p_{2}$ can successfully handle both cases of simulated datasets generated by GenGO model and NetGen model.

Table 1 The enrichment analysis result of NetGen on lung cancer dataset

\begin{tabular}{|c|c|c|c|}
\hline Rank & $\mathrm{GO}$ ID & Description & $p$-value \\
\hline 1 & GO:0006491 & $\mathrm{N}$-glycan processing & $1.32 \mathrm{e}-3$ \\
\hline 2 & GO:0006662 & glycerol ether metabolic process & $1.93 e-3$ \\
\hline 3 & GO:0006175 & dATP biosynthetic process & $5.54 \mathrm{e}-3$ \\
\hline 4 & GO:0060149 & negative regulation of posttranscriptional gene silencing & $5.54 \mathrm{e}-3$ \\
\hline 5 & GO:0006043 & glucosamine catabolic process & $5.54 \mathrm{e}-3$ \\
\hline 6 & GO:0035772 & interleukin-13-mediated signaling pathway & $5.54 \mathrm{e}-3$ \\
\hline 7 & GO:2000832 & negative regulation of steroid hormone secretion & $1.11 \mathrm{e}-2$ \\
\hline 8 & GO:0021648 & vestibulocochlear nerve morphogenesis & $1.11 \mathrm{e}-2$ \\
\hline 9 & GO:0072318 & clathrin coat disassembly & $1.11 \mathrm{e}-2$ \\
\hline 10 & GO:1900748 & positive regulation of vascular endothelial growth factor signaling pathway & $1.65 \mathrm{e}-2$ \\
\hline 11 & GO:0015014 & $\begin{array}{l}\text { heparan sulfate proteoglycan biosynthetic process, polysaccharide chain } \\
\text { biosynthetic process }\end{array}$ & $1.65 \mathrm{e}-2$ \\
\hline 12 & GO:0000710 & meiotic mismatch repair & $1.65 \mathrm{e}-2$ \\
\hline 13 & GO:0030070 & insulin processing & $1.65 \mathrm{e}-2$ \\
\hline 14 & GO:0072190 & ureter urothelium development & $1.65 \mathrm{e}-2$ \\
\hline 15 & GO:0034154 & toll-like receptor 7 signaling pathway & $2.20 \mathrm{e}-2$ \\
\hline 16 & GO:2000195 & negative regulation of female gonad development & 1 \\
\hline 17 & GO:0016082 & synaptic vesicle priming & 1 \\
\hline 18 & GO:2000370 & positive regulation of clathrin-dependent endocytosis & 1 \\
\hline 19 & GO:0002143 & tRNA wobble position uridine thiolation & 1 \\
\hline 20 & GO:0060978 & angiogenesis involved in coronary vascular morphogenesis & 1 \\
\hline
\end{tabular}

Best parameter setting: $p_{1}=0.5, p_{2}=0.05, q=0.001$. Term combination $p=1.50 \mathrm{e}-24$

An appropriate parameter combination identified via a mixed parameter selection strategy was shown at the bottom of the table. The Fisher's exact test $p$-values for single term and term combination were listed. The GO terms in bold were particularly identified by NetGen 


\section{Real data studies}

To test the efficiency of NetGen in real datasets, we used four GEO microarray gene expression profiles of human complex diseases to execute real data analysis. More details about the data description and the preprocessing can be found in Additional file 1 .

For each dataset, the dataset-specific active gene list was generated as introduced in the Methods section. Since the true parameter combination for generating active gene list from real datasets is unknown, we performed a mixed parameter selection strategy to obtain multiple solutions (see Methods). The evaluation of the mixed parameter selection strategy on the simulation studies showed that the generating parameters are not necessarily the best solving parameters (see Additional file 1 Figure S10-S11). Instead to infer the true values of the generative parameters, which is very difficult and may be meaningless, the mixed parameter selection strategy intends to produce multiple solutions in real applications, and reveal more information of the underlying biological processes for the downstream analysis. Due to the space limit, we only analyzed the enriched term set with the lowest combination $p$-value. The results of enrichment analysis are shown in Table 1, 2, 3 and 4 . The GO terms that were not identified by GenGO with same mixed parameter selection strategy are shown in bold.

As for lung cancer dataset, we obtained a significantly enriched term set, whose combination $p$-value computed by Fisher's exact test was $1.5 \times 10^{-24}$, including 20 lungrelated terms (Table 1). Notably, five terms (GO:2000195, GO:0016082, GO:2000370, GO:0002143, GO:0060978) with $p$-value equal to 1 were particularly identified by NetGen, i.e. these terms are not directly annotated to the active genes. However, these five terms discovered when taking the supplementary role of network information into consideration actually showed a closely relationship with lung tumorigenesis. For examples, mutations in clathrin and several of its associated proteins and adaptors (EPS15, HIP1, CALM, endophilin and $\beta$-arrestin 1) were identified via systematic characterization of somatic mutations in breast, renal and lung cancers [26, 27], which is the main function in term GO:2000370 (positive regulation of clathrin-dependent endocytosis). As for GO:0016082 (synaptic vesicle priming), exosome is closely related to synaptic vesicle cycle. In pathological states, such as cancer, a number of key proteins and microRNAs are expulsed due to the exosome-mediated abnormal activity of the export machinery $[28,29]$. For GO:0002143 (tRNA wobble position uridine thiolation), the aberrant expression of tRNA

Table 2 The enrichment analysis result of NetGen on ulcerative colitis dataset

\begin{tabular}{|c|c|c|c|}
\hline Rank & GO ID & Description & $p$-value \\
\hline 1 & GO:0032968 & positive regulation of transcription elongation from RNA polymerase II promoter & $1.10 \mathrm{e}-3$ \\
\hline 2 & GO:0018874 & benzoate metabolic process & $3.83 e-3$ \\
\hline 3 & GO:0010900 & negative regulation of phosphatidylcholine catabolic process & $3.83 e-3$ \\
\hline 4 & GO:1900402 & $\begin{array}{l}\text { regulation of carbohydrate metabolic process by regulation of transcription from RNA } \\
\text { polymerase II promoter }\end{array}$ & $3.83 e-3$ \\
\hline 5 & GO:0006294 & nucleotide-excision repair, preincision complex assembly & $3.83 e-3$ \\
\hline 6 & GO:0038193 & thromboxane A2 signaling pathway & $3.83 e-3$ \\
\hline 7 & GO:0031119 & tRNA pseudouridine synthesis & $7.65 e-3$ \\
\hline 8 & GO:0007439 & ectodermal digestive tract development & $7.65 e-3$ \\
\hline 9 & GO:0009240 & isopentenyl diphosphate biosynthetic process & $1.15 e-2$ \\
\hline 10 & GO:0045196 & establishment or maintenance of neuroblast polarity & $1.15 e-2$ \\
\hline 11 & GO:0006154 & adenosine catabolic process & $1.15 e-2$ \\
\hline 12 & GO:0002254 & kinin cascade & $1.15 e-2$ \\
\hline 13 & GO:2000681 & negative regulation of rubidium ion transport & $1.15 e-2$ \\
\hline 14 & GO:0035701 & hematopoietic stem cell migration & $1.52 \mathrm{e}-2$ \\
\hline 15 & GO:0008612 & peptidyl-lysine modification to peptidyl-hypusine & $1.52 \mathrm{e}-2$ \\
\hline 16 & GO:1901299 & negative regulation of hydrogen peroxide-mediated programmed cell death & 1 \\
\hline 17 & GO:1901841 & regulation of high voltage-gated calcium channel activity & 1 \\
\hline 18 & GO:0043547 ${ }^{\mathrm{a}}$ & positive regulation of GTPase activity & 1 \\
\hline \multicolumn{2}{|c|}{ Best parameter setting: $p_{1}=0.5, p_{2}=0.05, q=0.001$. } & Term combination $p=1.33 \mathrm{e}-27$ & \\
\hline
\end{tabular}

${ }^{\mathrm{a}} \mathrm{GO}: 0032850$ updated to alternate term GO:0043547

An appropriate parameter combination identified via a mixed parameter selection strategy was shown at the bottom of the table. The Fisher's exact test $p$-values for single term and term combination were listed. The GO terms in bold were particularly identified by NetGen 
Table 3 The enrichment analysis result of NetGen on cervical carcinogenesis dataset

\begin{tabular}{|c|c|c|c|}
\hline Rank & GO ID & Description & $p$-value \\
\hline 1 & GO:0006271 & DNA strand elongation involved in DNA replication & $3.42 \mathrm{e}-11$ \\
\hline 2 & GO:0090224 & regulation of spindle organization & $1.78 \mathrm{e}-3$ \\
\hline 3 & GO:0001927 & exocyst assembly & $6.43 e-3$ \\
\hline 4 & GO:0038016 & insulin receptor internalization & $6.43 e-3$ \\
\hline 5 & GO:0070676 & intralumenal vesicle formation & $6.43 e-3$ \\
\hline 6 & GO:0086042 & cardiac muscle cell-cardiac muscle cell adhesion & $6.43 e-3$ \\
\hline 7 & GO:0014738 & regulation of muscle hyperplasia & $1.28 \mathrm{e}-2$ \\
\hline 8 & GO:2000393 & negative regulation of lamellipodium morphogenesis & $1.28 \mathrm{e}-2$ \\
\hline 9 & GO:0010993 & regulation of ubiquitin homeostasis & $1.28 \mathrm{e}-2$ \\
\hline 10 & GO:0006050 & mannosamine metabolic process & $1.28 \mathrm{e}-2$ \\
\hline 11 & GO:0046602 & regulation of mitotic centrosome separation & $1.92 \mathrm{e}-2$ \\
\hline 12 & GO:0072708 & response to sorbitol & $1.92 \mathrm{e}-2$ \\
\hline 13 & GO:0001992 & regulation of systemic arterial blood pressure by vasopressin & $1.92 \mathrm{e}-2$ \\
\hline 14 & GO:1902498 & regulation of protein autoubiquitination & $1.92 \mathrm{e}-2$ \\
\hline 15 & GO:0048388 & endosomal lumen acidification & $1.92 \mathrm{e}-2$ \\
\hline 16 & GO:0048280 & vesicle fusion with Golgi apparatus & $2.55 e-2$ \\
\hline 17 & GO:0097264 & self proteolysis & $3.18 \mathrm{e}-2$ \\
\hline 18 & GO:0045329 & carnitine biosynthetic process & $3.18 \mathrm{e}-2$ \\
\hline 19 & GO:0051382 & kinetochore assembly & $7.45 e-2$ \\
\hline 20 & GO:0000741 & karyogamy & 1 \\
\hline 21 & GO:2000656 & regulation of apolipoprotein binding & 1 \\
\hline 22 & GO:0032848 & negative regulation of cellular $\mathrm{pH}$ reduction & 1 \\
\hline 23 & GO:0090158 & endoplasmic reticulum membrane organization & 1 \\
\hline
\end{tabular}

Best parameter setting: $p_{1}=0.5, p_{2}=0.05, q=0.001$. Term combination $p=1.10 \mathrm{e}-37$

An appropriate parameter combination identified via a mixed parameter selection strategy was shown at the bottom of the table. The Fisher's exact test $p$-values for single term and term combination were listed. The GO terms in bold were particularly identified by NetGen

modification plays an important role in complex diseases [30]. Besides, thiolation-based chemotherapy has been proposed for lung cancer [31].

The combination $p$-value of identified term set for ulcerative colitis dataset was $1.33 \times 10^{-27}$, including 18 disease-related terms (Table 2). Adding the network information assisted our model to identify three terms (GO:1901299, GO:1901841, GO:0043547, Fisher's exact test $p$-value $=1$ ), which have no directly connections with the active genes. These three terms showed closely relationships with carcinogenesis. For example, as for term GO:1901841 (regulation of high voltage-gated calcium channel activity), substantial researches showed that calcium channel intimately connected with cancer cells proliferation and metastasis [32, 33]. For GO:1901299 (negative regulation of hydrogen peroxide-mediated programmed cell death), hydrogen peroxide $\left(\mathrm{H}_{2} \mathrm{O}_{2}\right)$ plays a key role in tumorigenesis. Superfluous increasing of $\mathrm{H}_{2} \mathrm{O}_{2}$ generated by cancer cell may lead to several pivotal changes, such as DNA alteration, cell proliferation, apoptosis and angiogenesis, during tumorigenesis [34, 35].

For cervical carcinogenesis dataset, a set of 23 disease-related terms, whose combination $p$-value was $1.10 \times 10^{-37}$, were identified (Table 3). Among these identified terms, four terms (GO:0000741, GO:2000656, GO:0032848, GO:0090158), which had no directly overlap with the active genes, were recovered when adding the network information. Particularly, GO:2000656 is related to the regulation of apolipoprotein binding. Many studies showed that apolipoprotein took part in the tumor progression [36, 37]. The function of GO:0032848 (negative regulation of cellular $\mathrm{pH}$ reduction) is mainly connected with the regulation of intracellular acid-base. The particular mechanisms of $\mathrm{pH}$ sensing and regulation in tumor can be a common physical hallmark of solid tumors [38].

As for renal cell carcinoma dataset, the combination $p$-value of identified term set, including 27 terms, was $8.68 \times 10^{-50}$ (Table 4). Similar phenomena were also observed for the $p$-values of three terms (GO:0002542, 
Table 4 The enrichment analysis result of NetGen on renal cell carcinoma dataset

\begin{tabular}{|c|c|c|c|}
\hline Rank & GO ID & Description & $p$-value \\
\hline 1 & GO:0090259 & regulation of retinal ganglion cell axon guidance & $7.56 \mathrm{e}-7$ \\
\hline 2 & GO:0033572 & transferrin transport & $1.05 e-6$ \\
\hline 3 & GO:0072017 & distal tubule development & $3.03 e-5$ \\
\hline 4 & GO:2000054 & negative regulation of Wnt signaling pathway involved in dorsal/ventral axis specification & $3.34 \mathrm{e}-5$ \\
\hline 5 & GO:0072015 & glomerular visceral epithelial cell development & $1.17 e-3$ \\
\hline 6 & GO:2000287 & positive regulation of myotome development & $5.81 e-3$ \\
\hline 7 & GO:0006113 & fermentation & $5.81 e-3$ \\
\hline 8 & GO:0051460 & negative regulation of corticotropin secretion & $5.81 e-3$ \\
\hline 9 & GO:0060720 & spongiotrophoblast cell proliferation & $5.81 e-3$ \\
\hline 10 & GO:0043438 & acetoacetic acid metabolic process & $5.81 e-3$ \\
\hline 11 & GO:0032972 & regulation of muscle filament sliding speed & $5.81 e-3$ \\
\hline 12 & GO:0090038 & negative regulation of protein kinase $C$ signaling & $1.16 \mathrm{e}-2$ \\
\hline 13 & GO:0035425 & autocrine signaling & $1.16 \mathrm{e}-2$ \\
\hline 14 & GO:0010760 & negative regulation of macrophage chemotaxis & $1.16 \mathrm{e}-2$ \\
\hline 15 & GO:0060161 & positive regulation of dopamine receptor signaling pathway & $1.73 e-2$ \\
\hline 16 & GO:0097411 & hypoxia-inducible factor-1alpha signaling pathway & $1.73 e-2$ \\
\hline 17 & GO:0060435 & bronchiole development & $1.73 e-2$ \\
\hline 18 & GO:0051933 & amino acid neurotransmitter reuptake & $1.73 e-2$ \\
\hline 19 & GO:0046598 & positive regulation of viral entry into host cell & $2.31 e-2$ \\
\hline 20 & GO:0015015 & heparan sulfate proteoglycan biosynthetic process, enzymatic modification & $2.87 e-2$ \\
\hline 21 & GO:0006572 & tyrosine catabolic process & $2.87 e-2$ \\
\hline 22 & GO:0019532 & oxalate transport & $2.87 e-2$ \\
\hline 23 & GO:0072171 & mesonephric tubule morphogenesis & $3.44 \mathrm{e}-2$ \\
\hline 24 & GO:0051156 & glucose 6-phosphate metabolic process & $5.12 \mathrm{e}-2$ \\
\hline 25 & GO:0002542 & Factor XII activation & 1 \\
\hline 26 & GO:0010766 & negative regulation of sodium ion transport & 1 \\
\hline 27 & GO:0014858 & positive regulation of skeletal muscle cell proliferation & 1 \\
\hline
\end{tabular}

Best parameter setting: $p_{1}=0.5, p_{2}=0.05, q=0.001$. Term combination $p=8.68 \mathrm{e}-50$

An appropriate parameter combination identified via a mixed parameter selection strategy was shown at the bottom of the table. The Fisher's exact test $p$-values for single term and term combination were listed. The GO terms in bold were particularly identified by NetGen

GO:0014858 and GO:0010766) were equal to 1, which revealed the function of network information. For example, the function of GO:0010766 (negative regulation of sodium ion transport) is related to the transportation of sodium ion. The control of the ionic equilibrium is the major function of kidney [39]. The similar term was also identified by Tun $H W$ et al., when using the different expression profiles of clear cell renal cell carcinoma [40]. For GO:0002542 (Factor XII activation), the activity of Factor XII is closely connected with the phenomenon, known as the enhanced permeability and retention (EPR) effect, which has been observed to be universal in solid tumors for lipid and macromolecular agents [41].

The identified enriched terms with a higher similarity and redundancy often makes the researchers harder to obtain the underlying biological interpretations. In addition to the above enriched analysis, we test the redundancy of the enriched terms identified via NetGen. Here, the averaged semantic similarity score was used to measure the redundancy of the identified terms (see Methods). The results can be found in Figure 4, which showed that the averaged semantic similarity scores of NetGen and GenGO, two combination-based approaches, were far below than the scores of the Fisher's exact test on four datasets. Besides, the score of NetGen was around the mean score of the random distribution, which indicates the redundancy of these identified terms were nearly minimized. In conclusion, NetGen can effectively reduce the redundancy of the identified terms, which is helpful in the exploration of the underlying pathogenesis of complex diseases. 

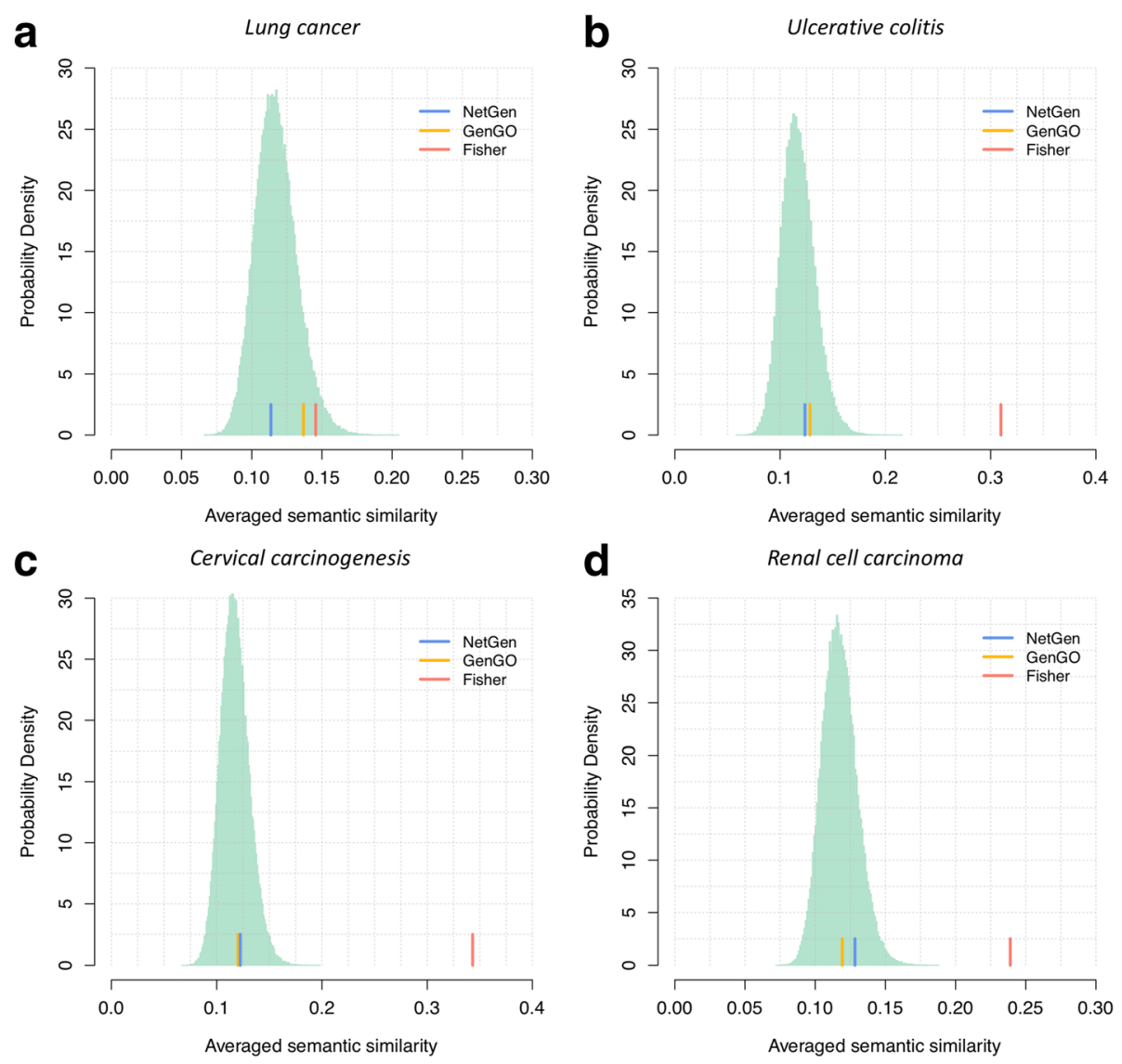

Fig. 4 Comparison of the averaged semantic similarity score in the identified term set. The light green distribution represents the semantic similarity score at the random level. The blue, orange and red bar represent the NetGen, GenGO and Fisher's exact test, respectively. The semantic similarity score was computed using the GOSemSim package [24] in $\mathrm{R}$

\section{Discussion}

The innovative component of NetGen is the integration of network information to extend the generative model of functional enrichment analysis. The framework of NetGen enables the users to exploit not only the PPI network but also various other distinct biomolecular networks, such as gene regulatory network, metabolic network and signal transduction network. Since different types of biomolecular networks reveal different levels and essential biological mechanisms in biological system, the selection of biomolecular network does have an influence on the performance of NetGen. Here, we did not compare the performance of NetGen with different biomolecular networks. One can select an appropriate network to assist the functional enrichment analysis according to the studied biological problem and available datasets. Another important point is the completeness or quality of the used network. The performance of NetGen would be greatly affected by the highly-noisy biological network since the wrong peripheral nodes due to noisy edges in the network will mess the results of enrichment analysis. Relatively speaking, the influence of the incomplete network may be small because the NetGen model will degenerate to some extent into the GenGO model. According to the parameter sensitivity analysis (see Additional file 1), it is better to choose a smaller $p_{2}$ when using a network with low quality.

The generative model is a computational model that assumes that the observed data was generated under certain probabilistic model and some distributions. The observed data was then used to estimate the parameters of the probabilistic model and distributions, and to infer values that could not be directly observed. Through maximizing a log-likelihood function, NetGen can identify the most likely significant terms. In this paper, we used a greedy approximation algorithm to seek for a near-optimal solution of the $0-1$ integer programming problem. Based on this greedy algorithm, the running time of NetGen, depending on the size of input annotation matrix, is acceptable for large datasets in real applications. On the other hand, the solution quality of functional enrichment analysis is also affected by the approximation algorithm. In this work, the 
performance of NetGen using different approximation algorithm was not compared, which will be one of the goals in our future research.

There are three main parameters, $p_{1}, p_{2}, q$, in our model, which were explained in the generative process. We executed the parameter sensitivity analysis (see Additional file 1) to test their robustness in related enrichment analysis. The sensitivity analysis result can be a reference to help the user selecting an appropriate parameter combination. Besides, one can obtain a more explicit and intuitive explanation from some special cases. If the parameters are set as $p_{1}=1, p_{2}=0, q=0$, the model is equivalent to identify the term set that the union of their directly annotated genes has the most overlap with the active gene list. Similarly, if the parameters are set as $p_{1}=1, p_{2}=1, q=0$, the annotated genes in one term should add the corresponding neighbor genes in biological network. From this perspective, NetGen is a generalization to the above simple enrichment strategy and unifies these model into one framework.

Usually the biologists will obtain one ranked gene list for functional enrichment analysis. For example, the differential expression genes are often ranked by the $\mathrm{t}$-test $p$-value. The rank information of gene list is not exploited in NetGen. It will be useful if the rank information of gene list can be considered and the outputs of functional enrichment analysis may become more precise. However, it is very difficult to integrate the rank information into the current term combination-based approaches. To the best of our knowledge, such kind of methods has not been studied in literature (see Additional file 1 Table S4). This will be one of the directions in our future research.

\section{Conclusions}

In this paper, a novel network-based probabilistic generative model, NetGen, was proposed to perform the enrichment analysis. An additional protein-protein interaction network was explicitly used to assist the functional enrichment analysis. NetGen achieved a superior performance than other compared methods in the simulation studies. Besides, several important GO terms, which were not directly linked with the active gene list, were exclusively identified by NetGen on real datasets.

In real applications, NetGen was not restrict on Homo sapiens but can also be applied on any other species. Our procedure leads to a more reasonable and explainable result of the functional enrichment analysis. As a novel term combination-based functional enrichment analysis method, NetGen is complementary to current individual term-based methods. We believed that NetGen is an efficient computational tool for functional enrichment analysis and can help to explore the underlying pathogenesis of complex diseases.

\section{Additional file}

Additional file 1: Supplementary materials including the classification of enrichment analysis methods, the parameter sensitivity analysis, the additional simulation results, and the description of gene expression datasets, GO annotation data, and active gene lists used in real data applications. (PDF $1385 \mathrm{~kb}$ )

\section{Abbreviations}

BP: Biological process; CC: Cellular component; FN: False negative; FP: False positive; GEO: Gene Expression Omnibus; GO: Gene ontology; IQP: Integer quadratic programming; MF: Molecular function; MLE: Maximum likelihood estimation; PPI: Protein-protein interaction; TP: True positive

\section{Acknowledgements}

Not applicable.

\section{Availability of data and materials}

The proposed method has been implemented in the $\mathrm{R}$ package CopTea publicly available at GitHub website, http://github.com/wulingyun/CopTea/. The datasets generated and analyzed during the current study are available in http://doc.aporc.org/wiki/NetGen.

\section{About this supplement}

This article has been published as part of BMC Systems Biology Volume 11 Supplement 4, 2017: Selected papers from the 10th International Conference on Systems Biology (ISB 2016). The full contents of the supplement are available online at https://bmcsystbiol.biomedcentral.com/articles/ supplements/volume-11-supplement-4.

\section{Funding}

This work was supported by the Strategic Priority Research Program of the Chinese Academy of Sciences [grant number XDB13040600]; and the National Natural Science Foundation of China [grant numbers 91330114 , 11131009, 11631014, 11661141019]. The publication cost was funded by the National Natural Science Foundation of China [grant number 91330114].

\section{Authors' contributions}

LYW designed the research. DS and YL carried out the experiments, wrote the program and analyzed the data. DS, YL and LYW drafted and revised the paper. All authors read and approved the final manuscript.

Ethics approval and consent to participate Not applicable.

\section{Consent for publication}

Not applicable.

\section{Competing interests}

The authors declare that they have no competing interests.

\section{Publisher's Note}

Springer Nature remains neutral with regard to jurisdictional claims in published maps and institutional affiliations.

\section{Author details}

${ }^{1}$ Institute of Applied Mathematics, Academy of Mathematics and Systems Science, Chinese Academy of Sciences, Beijing 100190, China. ${ }^{2}$ National Center for Mathematics and Interdisciplinary Sciences, Chinese Academy of Sciences, Beijing 100190, China. ${ }^{3}$ School of Mathematical Sciences, University of Chinese Academy of Sciences, Beijing 100049, China.

Published: 21 September 2017

\section{References}

1. Gene Ontology C. The gene ontology (GO) project in 2006. Nucleic Acids Res. 2006;34(Database issue):D322-6. 
2. Harris MA, Clark J, Ireland A, Lomax J, Ashburner M, Foulger R, Eilbeck K, Lewis S, Marshall B, Mungall C, et al. The gene ontology (GO) database and informatics resource. Nucleic Acids Res. 2004;32(Database issue):D258-61.

3. Ashburner M, Ball CA, Blake JA, Botstein D, Butler H, Cherry JM, Davis AP Dolinski K, Dwight SS, Eppig JT, et al. Gene ontology: tool for the unification of biology. The Gene Ontology Consortium. Nat Genet. 2000;25(1):25-9.

4. Zeeberg BR, Feng W, Wang G, Wang MD, Fojo AT, Sunshine M, Narasimhan S, Kane DW, Reinhold WC, Lababidi S, et al. GoMiner: a resource for biological interpretation of genomic and proteomic data. Genome Biol. 2003:4(4):R28.

5. Hosack DA, Dennis G Jr, Sherman BT, Lane HC, Lempicki RA. Identifying biological themes within lists of genes with EASE. Genome Biol. 2003;4(10):R70

6. Beissbarth T, Speed TP. GOstat: find statistically overrepresented gene Ontologies within a group of genes. Bioinformatics. 2004;20(9):1464-5.

7. Khatri P, Draghici S, Ostermeier GC, Krawetz SA. Profiling gene expression using onto-express. Genomics. 2002;79(2):266-70.

8. Glaab E, Baudot A, Krasnogor N, Schneider R, Valencia A. EnrichNet: networkbased gene set enrichment analysis. Bioinformatics. 2012;28(18):i451-7.

9. Doniger SW, Salomonis N, Dahlquist KD, Vranizan K, Lawlor SC, Conklin BR. MAPPFinder: using gene ontology and GenMAPP to create a global geneexpression profile from microarray data. Genome Biol. 2003;4(1):R7.

10. Fisher RA. On the interpretation of $x(2)$ from contingency tables, and the calculation of P. J R Stat Soc. 1922:85:87-94.

11. Subramanian A, Tamayo P, Mootha VK, Mukherjee S, Ebert BL, Gillette MA, Paulovich A, Pomeroy SL, Golub TR, Lander ES, et al. Gene set enrichment analysis: a knowledge-based approach for interpreting genome-wide expression profiles. Proc Natl Acad Sci U S A. 2005;102(43):15545-50.

12. Goeman JJ, van de Geer SA, de Kort F, van Houwelingen HC. A global test for groups of genes: testing association with a clinical outcome. Bioinformatics. 2004;20(1):93-9.

13. Tian L, Greenberg SA, Kong SW, Altschuler J, Kohane IS, Park PJ. Discovering statistically significant pathways in expression profiling studies. Proc Natl Acad Sci U S A. 2005;102(38):13544-9.

14. Kim SY, Volsky DJ. PAGE: parametric analysis of gene set enrichment. BMC Bioinformatics. 2005;6:144.

15. Barry WT, Nobel AB, Wright FA. Significance analysis of functional categories in gene expression studies: a structured permutation approach. Bioinformatics. 2005;21(9):1943-9.

16. Zhou X, Su Z. EasyGO: gene ontology-based annotation and functional enrichment analysis tool for agronomical species. BMC Genomics. 2007:8:246.

17. Huang DW, Sherman BT, Tan Q, Kir J, Liu D, Bryant D, Guo Y, Stephens R, Baseler MW, Lane HC, et al. DAVID bioinformatics resources: expanded annotation database and novel algorithms to better extract biology from large gene lists. Nucleic Acids Res. 2007;35(Web Server issue):W169-75.

18. Bauer S, Gagneur J, Robinson PN. GOing Bayesian: model-based gene set analysis of genome-scale data. Nucleic Acids Res. 2010;38(11):3523-32.

19. Lu Y, Rosenfeld R, Simon I, Nau GJ, Bar-Joseph Z. A probabilistic generative model for GO enrichment analysis. Nucleic Acids Res. 2008;36(17):e109.

20. Frost HR, McCray AT. Markov chain ontology analysis (MCOA). BMC Bioinformatics. 2012;13:23.

21. Wang J, Huang Q, Liu ZP, Wang Y, Wu LY, Chen L, Zhang XS. NOA: a nove network ontology analysis method. Nucleic Acids Res. 2011;39(13):e87.

22. Alexeyenko A, Lee W, Pernemalm M, Guegan J, Dessen P, Lazar V, Lehtio J Pawitan Y. Network enrichment analysis: extension of gene-set enrichment analysis to gene networks. BMC Bioinformatics. 2012;13:226.

23. Keshava Prasad TS, Goel R, Kandasamy K, Keerthikumar S, Kumar S, Mathivanan S, Telikicherla D, Raju R, Shafreen B, Venugopal A, et al. Human protein reference database-2009 update. Nucleic Acids Res. 2009; 37(Database issue):D767-72.

24. Yu G, Li F, Qin Y, Bo X, Wu Y, Wang S. GOSemSim: an R package for measuring semantic similarity among GO terms and gene products. Bioinformatics. 2010;26(7):976-8.

25. Gentleman RC, Carey VJ, Bates DM, Bolstad B, Dettling M, Dudoit S, Ellis B, Gautier L, Ge Y, Gentry J, et al. Bioconductor: open software development for computational biology and bioinformatics. Genome Biol. 2004:5(10):R80.

26. McMahon HT, Boucrot E. Molecular mechanism and physiological functions of clathrin-mediated endocytosis. Nat Rev Mol Cell Biol. 2011;12(8):517-33.

27. Kan Z, Jaiswal BS, Stinson J, Janakiraman V, Bhatt D, Stern HM, Yue P, Haverty PM, Bourgon R, Zheng J, et al. Diverse somatic mutation patterns and pathway alterations in human cancers. Nature. 2010;466(7308):869-73.
28. Azmi AS, Bao B, Sarkar FH. Exosomes in cancer development, metastasis, and drug resistance: a comprehensive review. Cancer Metastasis Rev. 2013; 32(3-4):623-42.

29. Sudhof TC. The synaptic vesicle cycle. Annu Rev Neurosci. 2004;27:509-47.

30. Torres AG, Batlle E. Ribas de Pouplana L: role of tRNA modifications in human diseases. Trends Mol Med. 2014;20(6):306-14.

31. Jiang $L$, Li X, Liu L, Zhang Q. Thiolated chitosan-modified PLA-PCL-TPGS nanoparticles for oral chemotherapy of lung cancer. Nanoscale Res Lett. 2013;8(1):66.

32. Bose T, Cieslar-Pobuda A, Wiechec $E$. Role of ion channels in regulating $\mathrm{ca}(2)(+)$ homeostasis during the interplay between immune and cancer cells. Cell Death Dis. 2015;6:e1648.

33. Rao VR, Perez-Neut M, Kaja S, Gentile S. Voltage-gated ion channels in cancer cell proliferation. Cancers. 2015;7(2):849-75.

34. Szatrowski TP, Nathan CF. Production of large amounts of hydrogen peroxide by human tumor cells. Cancer Res. 1991;51(3):794-8.

35. Lopez-Lazaro M. Dual role of hydrogen peroxide in cancer: possible relevance to cancer chemoprevention and therapy. Cancer Lett. 2007;252(1):1-8.

36. Bae SM, Lee CH, Cho YL, Nam KH, Kim YW, Kim CK, Han BD, Lee YJ, Chun HJ, Ahn WS. Two-dimensional gel analysis of protein expression profile in squamous cervical cancer patients. Gynecol Oncol. 2005;99(1):26-35.

37. Dieplinger $H$, Ankerst DP, Burges $A$, Lenhard $M$, Lingenhel A, Fineder $L$, Buchner $\mathrm{H}$, Stieber P. Afamin and apolipoprotein A-IV: novel protein markers for ovarian cancer. Cancer Epidemiol Biomarkers Prev. 2009;18(4):1127-33.

38. Damaghi M, Wojtkowiak JW, Gillies RJ. pH sensing and regulation in cancer. Front Physiol. 2013:4:370

39. Motzer RJ, Bander NH, Nanus DM. Renal-cell carcinoma. N Engl J Med. 1996; 335(12):865-75

40. Tun HW, Marlow LA, von Roemeling CA, Cooper SJ, Kreinest P, Wu K, Luxon BA, Sinha M, Anastasiadis PZ, Copland JA. Pathway signature and cellular differentiation in clear cell renal cell carcinoma. PLoS One. 2010;5(5):e10696.

41. Maeda H, Wu J, Sawa T, Matsumura Y, Hori K. Tumor vascular permeability and the EPR effect in macromolecular therapeutics: a review. J Control Release. 2000;65(1-2):271-84

\section{Submit your next manuscript to BioMed Central and we will help you at every step:}

- We accept pre-submission inquiries

- Our selector tool helps you to find the most relevant journal

- We provide round the clock customer support

- Convenient online submission

- Thorough peer review

- Inclusion in PubMed and all major indexing services

- Maximum visibility for your research

Submit your manuscript at www.biomedcentral.com/submit
) Biomed Central 